\title{
An extreme bias in the germ line of XY C57BL/6<->XY FVB/N chimaeric mice
}

\author{
G. R. MacGregor* \\ Center for Molecular Medicine, \\ Emory University School of Medicine, Atlanta, GA 30322, USA
}

Chimaeric analysis is a powerful method to address questions about the cell-autonomous nature of defects in spermatogenesis. Symplastic spermatids (sys) mice have a recessive mutation that causes male sterility due to an arrest in germ-cell development during spermiogenesis. Chimaeric mice were generated by aggregation of eightcell embryos from sys (FVB/N genetic background) and wild-type C57BL/6 (B6) mice to determine whether the male germ-cell defect is cell-autonomous. The resulting FVB/N<->B6 chimaeras (<-> denotes fusion of embryos) were mated with $\mathrm{FVB} / \mathrm{N}$ mice and coat colour of offspring was used to identify transmission of $\mathrm{FVB} / \mathrm{N}$ or $\mathrm{B} 6$ gametes. Regardless of the relative contribution of $\mathrm{B} 6$ to somatic tissues of the chimaeras, almost all (282 of $284 ; 99.3 \%)$ offspring of B6 XY<->XY FVB/N (+/+ or sys/+) males $(n=9)$ received a $\mathrm{FVB} / \mathrm{N}$-derived paternal gamete. After mating of female $B 6<->F V B / N$ chimaeras, 51 of 73 $(69.9 \%)$ offspring received an FVB-derived maternal gamete. Southern blot analysis of different tissues from chimaeric males indicated that, despite the presence of balanced chimaerism in somatic tissues, the germ line in B6 $X Y<->X Y ~ F V B / N$ mice was essentially $F V B / N$ in composition. Thus there is a strong selective advantage for $\mathrm{FVB} / \mathrm{N}$ male germ cells over $\mathrm{B} 6$ male germ cells in $B 6<->F V B / N$-aggregation chimaeras at some stage during development of the male germ line. Each of three male chimaeras that were either $\mathrm{B} 6 \mathrm{XY}<->\mathrm{XY} \mathrm{FVB} / \mathrm{N}$ (sys/sys) or B6 $\mathrm{XX}<->\mathrm{XY} \mathrm{FVB} / \mathrm{N}$ (sys/sys) in composition was sterile, and testis histology was essentially sys mutant. This finding indicates that the function of the gene(s) affected in the sys mutation may be required in the testis, although whether expression is required in germ cells, somatic cells or both remains unknown. The extreme bias in transmission of male gametes has implications for experimental design in studies that use chimaeric analysis to address questions regarding the cell-autonomous nature of germ-cell defects in mice.

\section{Introduction}

Mammalian spermatogenesis involves the proliferation and differentiation of stem-cell spermatogonia that progress through meiosis as spermatocytes before undergoing terminal differentiation as haploid spermatids. During this process, germ-cell development is supported through intimate contact with the Sertoli cell, the somatic component of the seminiferous epithelium. Genetic defects can cause abnormalities in development of male germ cells. In such cases it can be difficult to determine whether the type of cell that is primarily affected by the mutation is the germ cell or a somatic cell. Chimaeric analysis is a powerful experimental method that can be used to determine whether a developmental defect is due to loss of gene function within the cell displaying the defect (that is, a cellautonomous defect), or is the result of an indirect effect of

*Present address: Center for Molecular and Mitochrondrial Medicine and Genetics, University of California, Irvine, CA 92697-2300, USA

Email: gmacg@uci.edu loss of the gene function in another type of cell (that is, a non-cell-autonomous defect) (Rossant and Spence, 1998). Historically, chimaeric studies in mice were first performed by aggregation of pairs of pre-compacted, preimplantationstage embryos (Tarkowski, 1961). This method has been used to investigate the male germ cell-autonomous nature of several factors, including influences on sperm morphology and fertilizing capacity (Burgoyne, 1975; Krzanowska et al., 1991) as well as genetic defects in spermatogenesis (Handel et al., 1987). Studies involving a chimaeric analysis of testis function usually require production of a relatively large number of animals because of the $25 \%$ probability that any aggregated embryo will have been derived from a fusion of two XY embryos. In such cases, cells from either embryo can, in theory, contribute to development of the testis, including the male germ line. In contrast, although $\mathrm{XY}<->\mathrm{XX}$ chimaeras (<-> denotes fusion of embryos; Mintz, 1964) usually develop as phenotypic males, the postnatal germ line is formed exclusively from the $X Y$ component and Sertoli cells are almost always $X Y$-derived. Thus, study of $\mathrm{XY}<->\mathrm{XX}$ chimaeras is uninformative when attempting to discriminate between a germ cell or Sertoli cell defect. An important premise of the experimental use of aggregation 
chimaeras is that a mixture of cells from each $X Y$ embryo will be found within each tissue in the adult animal.

Symplastic spermatids (sys) mice have a recessive mutation that results in male sterility (MacGregor et al., 1990). During the first wave of spermatogenesis in sys mutants there is a developmental arrest between steps 6 and 8 of spermiogenesis, when clones of developing germ cells lose contact with the supporting Sertoli cells and the intercellular bridges linking the spermatids open prematurely (MacGregor et al., 1990; Russell et al., 1991). The gene defect responsible for this mutant phenotype has not yet been identified. It is unclear whether the function of the gene(s) affected by the sys mutation is required in the testis, and if so, in which type of testicular cell expression is required for normal spermatogenesis. Chimaeric mice were generated by aggregation of sys homozygous or control (+/+, sys/+) embryos on an FVB/N strain background and wild-type embryos on a C57BL/6 (B6) strain background to determine whether the germ-cell defect in sys homozygous males is germ cell-autonomous. The resulting chimaeric males were mated to determine whether homozygous sys germ cells undergo normal development in a testicular environment containing wild-type somatic cells.

\section{Materials and Methods}

\section{Mice and superovulation}

Inbred male and female C57BL/6 $\left(\mathrm{a} / \mathrm{a},+^{B} /+^{B},+^{\left.\text {Tyr-C } /+{ }^{T y r}-\mathrm{C}\right)}\right.$ mice were purchased from Harlan (Indianapolis, IN). Inbred FVB/N $\left(+^{A} /+^{A},+{ }^{B} /+^{B}\right.$, Tyr-C/ Tyr-C) mice and ICR female mice were purchased from the National Cancer Institute. Mice were maintained on a $14 \mathrm{~h}$ light cycle (on 06:00 h, off 20:00 h). For superovulation, 4-6-week-old mice were injected i.p. with 5 iu equine chorionic gonadotrophin (Calbiochem, La Jolla, CA) at 14:00 h on day 0. Mice were subsequently administered i.p. with 5 iu hCG (Sigma, St Louis, MO) at 12:00 h on day 2, after which females were caged with stud males. Pseudopregnant female recipients were generated by mating ICR females that were in oestrus with vasectomized B6D2F1 stud males. All studies were conducted in accordance with guidelines provided by the AMVA and a protocol approved by an Institutional Animal Care and Use Committee.

\section{Production of chimaeric mice}

Chimaeric mice were generated using the method described by Hogan et al. (1994). In brief, uteri were excised from superovulated and mated females at day 2.5 after mating (12:00 h of the day on which a copulation plug was observed was designated as day 0.5 after mating). Eight-cell embryos were flushed from the proximal end of uteri using a $1 \mathrm{ml}$ syringe filled with BMOC-3 media (Invitrogen Corp., Carlsbad, CA) attached to a 33 g needle. Embryos were collected immediately and incubated in 50 $\mu$ pre-equilibrated BMOC-3, under paraffin oil, in $5 \% \mathrm{CO}_{2}$ in air at $37^{\circ} \mathrm{C}$. Zonae pellucidae were removed using acidified Tyrode's solution, followed by four washes in pre- equilibrated BMOC-3. Pairs of embryos were aggregated by making small depressions in a bacterial-grade plastic Petri dish (Sterilin, Stone) using the end of a pair of watchmaker forceps (Dumont, No. 5). Ten microlitres of BMOC-3 was added to each depression; the dish was covered with paraffin oil and pre-equilibrated at $37^{\circ} \mathrm{C}$, in $5 \% \mathrm{CO}_{2}$ in air. One healthy embryo from each strain was placed in each depression and the embryos were pushed carefully together. After incubation for $1 \mathrm{~h}$, the dish was removed gently from the incubator and examined. Any embryos that had separated were pushed together and the dish was incubated overnight at $37^{\circ} \mathrm{C}$, in $5 \% \mathrm{CO}_{2}$ in air in a humidified incubator. It was important to minimize the time that the embryos were out of the incubator. The next morning, aggregated embryos, which had usually developed a small blastocoel, were transferred surgically to the uteri of pseudopregnant females that had been anaesthetized (2,2,2-tribromoethanol (Avertin); Aldrich, Milwaukee, WI) as described by Hogan et al. (1994) at day 2.5 after mating. Eight embryos were transferred per uterine horn.

\section{Mating}

Chimaeric animals were mated beginning at 8 weeks of age. Each chimaeric male was mated in a trio, with two $\mathrm{FVB} / \mathrm{N}$ females, and each chimaeric female was mated with a single FVB/N male.

\section{Southern blot analysis}

Routine DNA sub-cloning was performed as described by Ausubel et al. (1994). DNA was extracted from tail, spleen or testis and Southern blot analysis was carried out as described by MacGregor et al. (1990). Paired testes from fully masculinized male chimaeras have almost identical contribution of tissue from each parental type (Gearhart and Oster-Granite, 1981; Handel et al., 1987), so it was assumed that histology and DNA composition would be similar between paired testes. The plasmid pZap1 contains a piece of mouse-genomic DNA from the sys locus (MacGregor et al., 1990). Plasmid pDP1171 (Mardon et al., 1989), which contains a region of the mouse Zfy 1 locus, was the kind gift of D. Page (Whitehead Institute, Boston, MA). Southern blot hybridization was conducted as described by MacGregor et al. (1990). All hybridization filters were washed to a final stringency of $\times 0.1$ standard saline citrate, $1 \%(\mathrm{w} / \mathrm{v})$ sodium dodecyl sulphate at $65^{\circ} \mathrm{C}$ before being exposed to Kodak XAR-5 film with an intensifying screen at $-80^{\circ} \mathrm{C}$.

\section{Histology}

Testes were excised and measured (pole-pole) using a Vernier calliper gauge before fixing by immersion in $15 \mathrm{ml}$ of Bouin's fluid (15 parts saturated picric acid:five parts $37 \%$ formaldehyde: one part glacial acetic acid) for $2 \mathrm{~h}$ at $4^{\circ} \mathrm{C}$. Testes were then removed, and the poles (approximately $10 \%$ at each end) were removed using a microtome 
blade and re-immersed in Bouin's fluid for a further 16-24 h at $4^{\circ} \mathrm{C}$. After dehydration in ethanols, clearing in xylenes and infiltration with paraffin wax (Paraplast Plus; Oxford Labware, St Louis, MO), testes were embedded in paraffin wax and sectioned at $5 \mu \mathrm{m}$. Sections were stained with periodic acid-Schiff-haematoxylin, mounted with a coverslip and viewed using a compound microscope equipped with a digital camera (SPOT; Diagnostic Instruments Inc., Sterling Heights, MI).

\section{Results}

\section{Experimental strategy}

The aim of the present study was to generate $X Y<->X Y$ male chimaeras that were derived from fusion of a sys homozygous FVB/N and a wild-type B6 eight-cell embryo. Sys heterozygous mutant females were superovulated and mated with sys heterozygous males to generate the sys embryos. Similarly, B6 females were superovulated and mated with B6 stud males. One in four embryos from the sys mating were expected to be sys homozygous and half of the embryos from both matings were expected to be male. Therefore, the incidence of sys homozygous B6 $\mathrm{XY}<->\mathrm{XY}$ $\mathrm{FVB} / \mathrm{N}$ animals should be one in 16 . Consequently, a target was set to produce at least 25 chimaeras for analysis, at least one of which was expected to consist of the desired genetic composition.

\section{Production of chimaeric mice}

Twenty-one male and six female chimaeric mice were generated. Dorsal views of each male and one female are shown (Fig. 1). FVB/N animals are albino and have white fur, whereas B6 mice have black coats. The fur of the chimaeras is composed of black, agouti and white hairs. Agouti pigmentation occurs in hair where B6 and FVB/N melanocytes are found in the same hair follicle (Mintz and Palm, 1969). The chimaeras displayed contributions from both parental strains. Approximately half of the chimaeras displayed balanced somatic chimaerism, as evidenced by their coat colour. After a breeding analysis, chimaeras were killed for molecular and histological analysis at 5 months of age.

\section{Molecular analysis of chimaeric mice}

Identification of $X Y<->X Y$ chimaeras. In theory, the testes of $X Y<->X Y$ male chimaeric mice are composed of a mixture of somatic and germ cells derived from each of the two strains used to generate the chimaeras. $X Y<->X X$ chimaeras normally develop as phenotypic male mice and can display balanced chimaerism in somatic tissues (Mystkowska and Tarkowski, 1968; McLaren, 1975; Gearhart and Oster-Granite, 1981; Krzanowska et al., 1991). However, XX spermatogonia fail to proliferate after birth owing to the absence of genes on the $Y$ chromosome, and the testis is composed exclusively of $X Y$ germ cells (for a review, see McLaren, 1983). The present study required the production of male chimaeras with a mixture of male germ cells within the testis, so it was important to discriminate male $X Y<->X Y$ chimaeras from male $X Y<->X X$ chimaeras. The $Y$ chromosome of $\mathrm{FVB} / \mathrm{N}$ mice is derived from Mus musculus domesticus, whereas that of B6 mice comes from M. musculus musculus (Mardon et al., 1989). A Taq I restriction fragment length polymorphism (RFLP) at the Zfy 1 locus can be used to discriminate these chromosomes (Mardon et al., 1989). Genomic DNA was purified from tail biopsies of chimaeric and control (non-chimaeric) mice and these were analysed by Southern blot hybridization to identify $X Y<->X Y$ chimaeric males. DNAs were digested with $T a q \mathrm{I}$, and the fragments were subjected to agarose gel electrophoresis and, after Southern blotting, were hybridized with a $1.6 \mathrm{~kb}$ Taq I-ECoRI fragment isolated from pDP1171 (Mardon et al., 1989). This probe detects bands of 9.0 and $4.5 \mathrm{~kb}$ specific for the M. m. musculus (B6) $\mathrm{Y}$ chromosome and 7.0 and $5.5 \mathrm{~kb}$ for the M. m. domesticus (FVB/N) Y chromosome. The results indicate that of the 21 male chimaeras analysed, 12 were $X Y<->X Y$, four were $X X(B 6)<->X Y(F V B / N)$ and five were $X Y(B 6)<->X X(F V B / N)$ (Fig. 2a; Table 1).

Genotyping at the sys locus. The Southern blot (Fig. 2a) was stripped and re-probed with a portion of genomic DNA flanking the transgene complex integration site in the sys mice to determine the genotype of the FVB/N (sys) component of these chimaeras (MacGregor et al., 1990). In conjunction with Taq I digestion, this probe permits discrimination of wild-type (B6), wild-type (FVB/N) and sys $(\mathrm{FVB} / \mathrm{N})$ alleles of chromosome 14 . The results of the analysis are shown (Fig. 2b) and summarized (Table 1). Of the 21 male chimaeras analysed, four were wild type, 12 heterozygous mutant sys and four homozygous mutant sys. On the basis of the Southern blot hybridization (Fig. 2b), and subsequent testis histology, one animal (1207) appeared to have been derived from more than two embryos, one of which was sys/sys. Consequently, chimaera 1207 was excluded from further analysis.

\section{Mating of chimaeras}

Chimaeric males and females were mated with FVB/N mice and the coat colour of the progeny was examined to determine the gametic contribution from the chimaeric parent. FVB/N inbred mice are albino (Taketo et al., 1991). Consequently, a zygote derived from fusion of two FVB/N gametes will produce albino progeny, whereas fusion of $\mathrm{FVB} / \mathrm{N}$ and B6 gametes will produce offspring with agouti fur. All nine (B6) $X Y<->X Y(F V B / N ;+/+$ or $+/$ sys) males sired progeny (Table 1). Neither of the two $X Y<->X Y$ animals that had a sys/sys component, nor the single B6 XX<->XY FVB (sys/sys) male produced progeny, despite mating with several different females over 4 months. In contrast to a previous report (Patek et al., 1991), the fecundity of $X Y<->X X$ sex-chimaeric males was not significantly different from that of $X Y<->X Y$ chimaeras (Table 2).

The coat colour of progeny from matings of $X Y<->X Y$ 


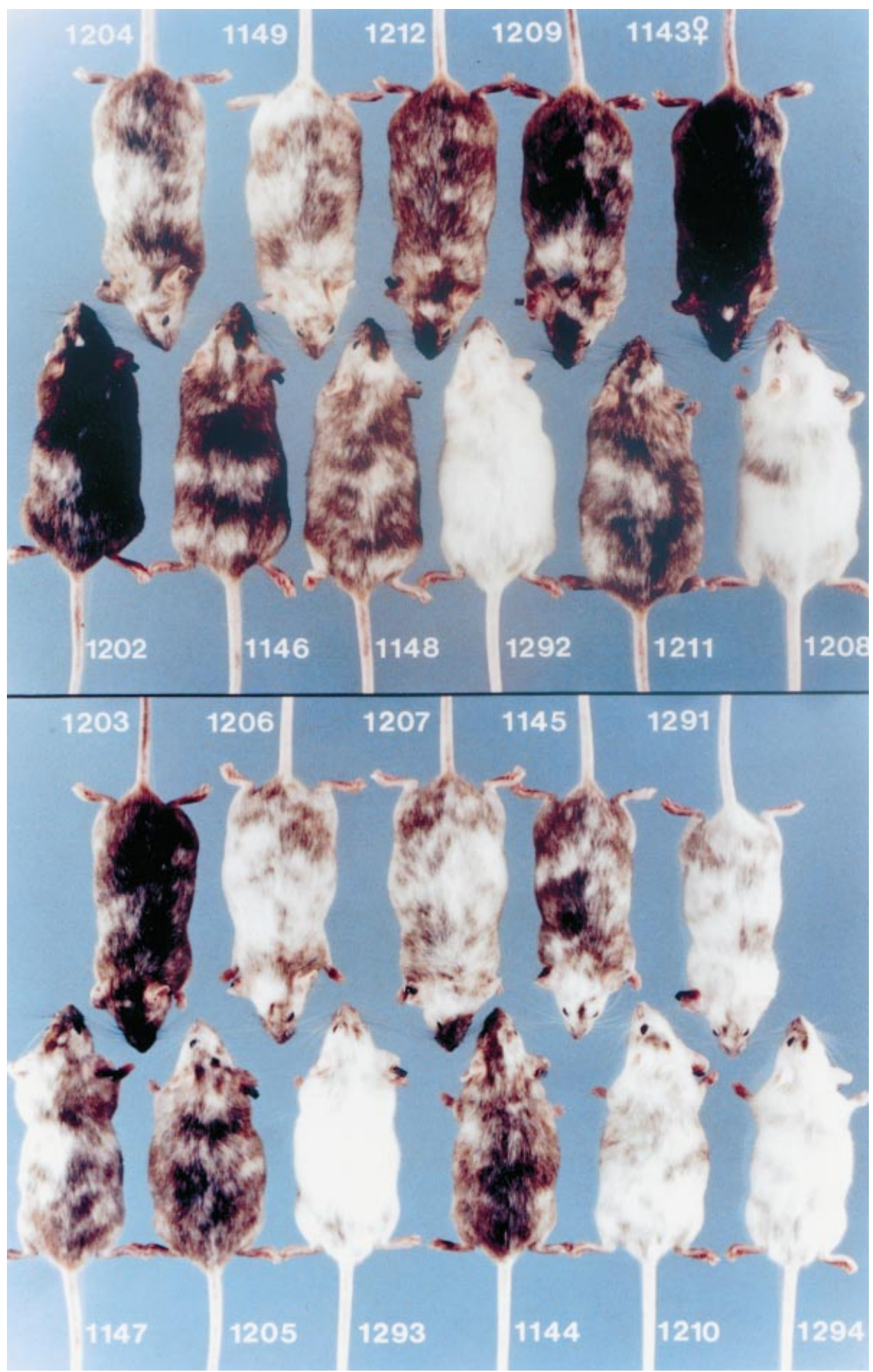

Fig. 1. Chimaeric mice used in the present study. Dorsal views of 21 male and one female (1143, upper right) chimaeric mice. Animals were anaesthetized before photography. On the basis of coat colour, approximately half of the animals display balanced somatic chimaerism.

male chimaeras, containing either a $+/+$ or $+/$ sys $\mathrm{FVB} / \mathrm{N}$ component, to FVB/N females was anticipated to be either albino or agouti, indicating transmission of $\mathrm{FVB} / \mathrm{N}$ or $\mathrm{B} 6$ gametes, respectively, from the chimaera. Surprisingly, of 284 offspring, only two had agouti coat colour, indicating an extremely low (approximately $0.7 \%$ ) rate of transmission 
(a)

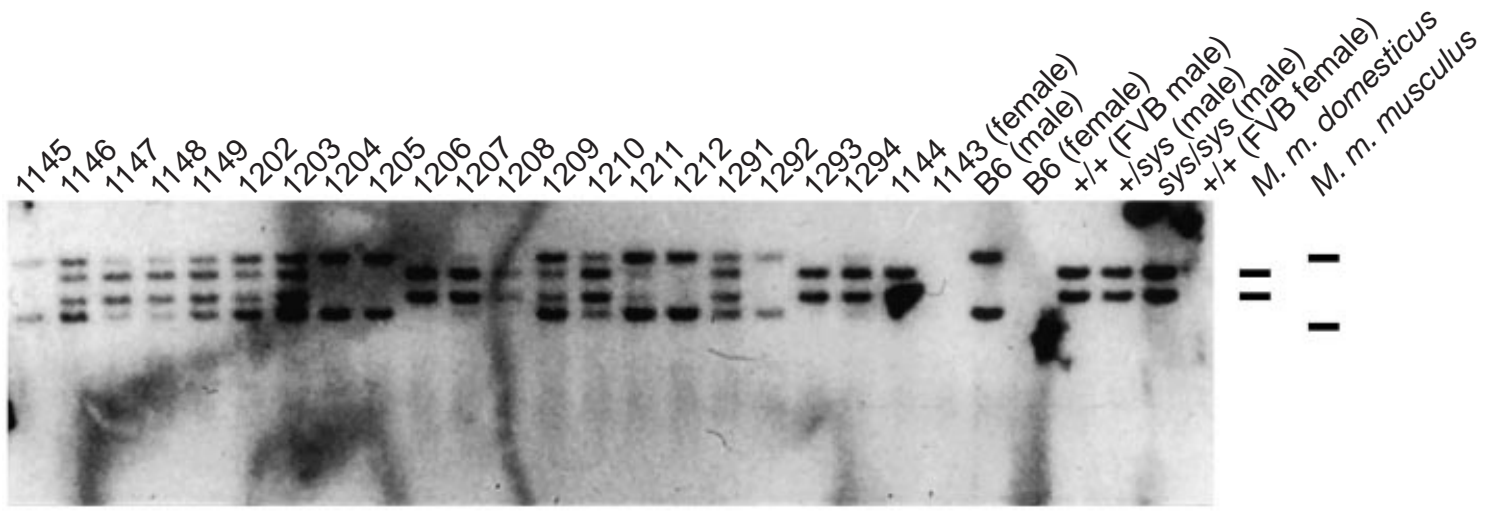

(b)

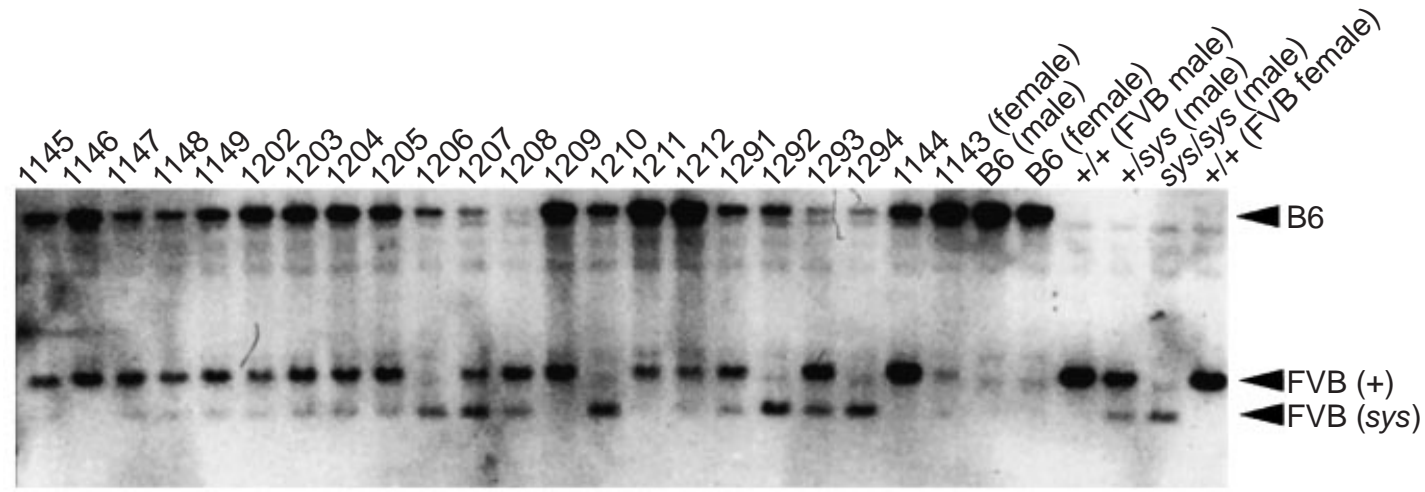

Fig. 2. Molecular genetic analysis of $\mathrm{FVB} / \mathrm{N}<->\mathrm{B} 6$ chimaeric mice. (a) Southern blot analysis of $\mathrm{Y}$-chromosome content of tail DNA from chimaeras. The blot was hybridized with a probe specific for the Y-linked Zfy 1 locus. The graphic on the right-hand side illustrates the hybridization patterns indicative of the presence of a Mus musculus domesticus (FVB/N) Y chromosome or a M. m. musculus (B6) Y chromosome. Lanes with numbers represent individual chimaeras. Control samples are shown on the right. (b) Southern blot analysis of tail DNA to determine genotype at sys locus of FVB/N component of chimaeras. The blot in (a) was stripped and re-hybridized with a probe from pZap1 (MacGregor et al., 1990), which identifies one flank of the transgene integration site in the sys allele on chromosome 14 (MacGregor et al., 1990). This probe detects a $2.3 \mathrm{~kb}$ band associated with the wild-type FVB allele (FVB (+)), a $0.9 \mathrm{~kb}$ band associated with the sys mutant FVB allele (FVB (sys)) and a $14 \mathrm{~kb}$ band associated with the C57BL/6 wild type allele (B6). The $0.9 \mathrm{~kb}$ band is relatively weak owing to deletion of much of the probe target sequences in the sys allele. Lanes with numbers represent individual chimaeras. Control samples are shown on the right. In chimaera 1145, the $0.9 \mathrm{~kb}$ (sys allele) band is extremely faint. However, confirmation of this genotype was made (Fig. 4). In chimaera 1207, the relative intensity of the FVB specific alleles ( $2.3 \mathrm{~kb}$ and $0.9 \mathrm{~kb})$ is inverted when compared with chimaeras with a sys/+ component, indicating that the animal may have arisen from aggregation of more than two embryos, two of which were B6 XY and FVB (sys/sys) XY. Consequently, this animal was not considered further.

of B6 gametes from these males. In contrast, breeding analysis of five chimaeric females that were presumed to be $\mathrm{XX}<->\mathrm{XX}$ produced progeny with a less biased contribution of B6 (30.1\%) and FVB/N (69.9\%) gametes. As expected, the offspring of male $X Y<->X X$ chimaeras inherited a gametic contribution exclusively from the $X Y$ component of the chimaera.

\section{Testis histology of chimaeras}

After completion of the mating analysis, chimaeric males were killed. Genomic DNA was prepared from the spleen as a source of somatic tissue and one testis as a source of (predominantly) germ line tissue. The contralateral testis was fixed in Bouin's fluid for histological examination. Testes from $X Y<->X Y$ chimaeras were mostly of a similar size, equivalent to those from non-chimaeric mice (Table 1). The exceptions were the two chimaeras that had an $X Y$ (sys/sys) component (chimaeras 1210 and 1294), the reduced testes sizes of which were similar to those in sys/sys (non-chimaeric) animals. Testes from $X Y<->X X$ chimaeras were either slightly smaller or similar to those in wild-type mice, with two exceptions. These were chimaera 1206, which contained an XY FVB (sys/sys) component, and 
Table 1. Breeding analysis of $\mathrm{FVB} / \mathrm{N}($ sys $)<->\mathrm{C} 57 \mathrm{BL} / 6$ mice

\begin{tabular}{|c|c|c|c|c|c|c|c|c|c|c|c|c|}
\hline $\begin{array}{l}\text { ID } \\
\text { number }\end{array}$ & B6 Y & FVB Y & $+/+$ & $+/$ sys & sys/sys & $\begin{array}{c}\text { Testicular } \\
\text { size }^{\mathrm{a}}\end{array}$ & $\begin{array}{l}\text { Percentage of } \\
\text { aspermatogenic } \\
\text { tubules }(n)\end{array}$ & $\begin{array}{l}\text { Expected } \\
\text { coat colour } \\
\text { of progeny }\end{array}$ & Litters & $\begin{array}{c}\text { Total } \\
\text { progeny }\end{array}$ & $\begin{array}{l}\text { Number } \\
\text { of albino }\end{array}$ & $\begin{array}{l}\text { Number } \\
\text { of agouti }\end{array}$ \\
\hline \multicolumn{13}{|c|}{ Phenotypic males } \\
\hline 1146 & $\bullet$ & $\bullet$ & $\bullet$ & & & 11.5 & $0.0(128)$ & Albino and agouti & 4 & 33 & 33 & 0 \\
\hline 1209 & - & - & $\bullet$ & & & 11.0 & $0.0(173)$ & Albino and agouti & 5 & 31 & 31 & 0 \\
\hline 1211 & $\bullet$ & $\bullet$ & $\bullet$ & & & 10.5 & $0.0(138)$ & Albino and agouti & 5 & 34 & 34 & 0 \\
\hline 1147 & - & - & & $\bullet$ & & 12.0 & $0.0(190)$ & Albino and agouti & 6 & 50 & 49 & 1 \\
\hline 1148 & $\bullet$ & $\bullet$ & & $\bullet$ & & 11.0 & $0.0(162)$ & Albino and agouti & 5 & 40 & 40 & 0 \\
\hline 1149 & $\bullet$ & - & & - & & 11.5 & $0.0(163)$ & Albino and agouti & 4 & 24 & 24 & 0 \\
\hline 1202 & $\bullet$ & $\bullet$ & & $\bullet$ & & 10.5 & $0.0(179)$ & Albino and agouti & 5 & 33 & 32 & 1 \\
\hline 1203 & $\bullet$ & - & & $\bullet$ & & 10.5 & $0.0(88)$ & Albino and agouti & 1 & 8 & 8 & 0 \\
\hline 1207 & $\bullet$ & - & $\bullet+$ & $\bullet+$ & - & ND & ND & ND & ND & ND & ND & ND \\
\hline 1291 & $\bullet$ & $\bullet$ & & $\bullet$ & & 10.5 & $2.6(114)$ & Albino and agouti & 4 & 31 & 31 & 0 \\
\hline 1210 & $\bullet$ & $\bullet$ & & & $\bullet$ & 6.0 & ND & ? & 0 & 0 & 0 & 0 \\
\hline 1294 & $\bullet$ & $\bullet$ & & & $\bullet$ & 6.5 & ND & $?$ & 0 & 0 & 0 & 0 \\
\hline 1144 & & - & $\bullet$ & & & 10.0 & $0.0(153)$ & Albino only & 3 & 34 & 34 & 0 \\
\hline 1208 & & $\bullet$ & & - & & 9.5 & $0.0(175)$ & Albino only & 4 & 26 & 26 & 0 \\
\hline 1293 & & - & & - & & 10.0 & $0.0(188)$ & Albino only & 5 & 38 & 38 & 0 \\
\hline 1206 & & $\bullet$ & & & $\bullet$ & 6.0 & ND & ? & 0 & 0 & 0 & 0 \\
\hline 1145 & $\bullet$ & & & $\bullet$ & & 9.0 & $34.4(128)$ & Agouti only & 3 & 15 & 0 & 15 \\
\hline 1204 & $\bullet$ & & & $\bullet$ & & 9.0 & $73.4(192)$ & Agouti only & 2 & 17 & 0 & 17 \\
\hline 1205 & $\bullet$ & & & $\bullet$ & & 9.5 & $1.9(154)$ & Agouti only & 3 & 25 & 0 & 25 \\
\hline 1212 & $\bullet$ & & & $\bullet$ & & 10.0 & $2.4(210)$ & Agouti only & 4 & 34 & 0 & 34 \\
\hline 1292 & $\bullet$ & & & & $\bullet$ & 5.5 & ND & Agouti only & 4 & 21 & 0 & 21 \\
\hline \multicolumn{13}{|c|}{ Phenotypic females } \\
\hline 1143 & & & $\bullet$ & & & NA & NA & Albino and agouti & 2 & 19 & 12 & 7 \\
\hline 1230 & & & ND & & & $\mathrm{NA}$ & NA & Albino and agouti & 2 & 19 & 6 & 13 \\
\hline 1231 & & & ND & & & NA & $\mathrm{NA}$ & Albino and agouti & 1 & 9 & 8 & 1 \\
\hline 1232 & & & ND & & & NA & NA & Albino and agouti & 1 & 10 & 10 & 0 \\
\hline 1233 & & & ND & & & NA & NA & Albino and agouti & 1 & 10 & 9 & 1 \\
\hline 1234 & & & ND & & & NA & $\mathrm{NA}$ & Albino and agouti & 1 & 6 & 6 & 0 \\
\hline
\end{tabular}

B6 Y: B6 male embryo; FVB Y: FVB/N male embryo; +/+, +/sys, sys/sys: genotype of FVB/N embryo at sys locus; expected coat colour of progeny: based upon transmission of FVB/N (albino)- or B6 (agouti)-derived gametes; litters: number of litters generated by chimaera; ND: not determined; NA: not applicable. aTesticular size: testes length (pole-pole, $\mathrm{mm})$; ?: experimental outcome is dependent on whether sys mutant gem cells have cell-autonomous defect; $(\bullet)$ : indicates the presence of a Y chromosome from either B6 and/or FVB/N, as well as the genotype of the FVB/N component at the sys locus. $(\bullet+)$ : based on the Southern blot analysis in Fig. 2b, chimaera No. 1207 appeared to have been derived from more than two embryos, which included B6 XY and FVB XY $($ sys/sys), with the identity of the other uncertain; consequently, this chimaera was not considered for subsequent analysis.

Table 2. Comparison of fecundity of $\mathrm{FVB} / \mathrm{N}($ sys $)<->\mathrm{C} 57 \mathrm{BL} / 6$ mice

\begin{tabular}{lcccc}
\hline Category & $\begin{array}{c}\text { Chimaera } \\
\text { composition }\end{array}$ & $\begin{array}{c}\text { Number of } \\
\text { animals }\end{array}$ & $\begin{array}{c}\text { Number of } \\
\text { litters }\end{array}$ & $\begin{array}{c}\text { Number of progeny } \\
\text { per litter (mean } \pm \text { SEM) }\end{array}$ \\
\hline A & B6 XY<->XY FVB & 9 & 39 & $7.26 \pm 0.34$ \\
B & B6 XX<->XY FVB & 3 & 12 & $8.17 \pm 0.75$ \\
C & B6 XY<->XX FVB & 5 & 16 & $7.00 \pm 0.71$ \\
\hline
\end{tabular}

Comparison of mean number of progeny per litter: categories $A$ and $B, t(10)=0.39, P>0.05$; categories $B$ and $C$, $t(6)=0.38, P>0.05$; categories $A$ and $C, t(12)=0.07, P>0.05$.

Data in this table were derived from chimaeras with XY FVB/N components that were either $+/+$ or $s y s /+$, that is, functionally wild type for spermatogenesis.

chimaera 1292 (B6 XY<->XX FVB sys/sys), the testes of which were approximately half the normal size. Chimaera 1292 was of interest because despite a relatively small proportion of XY cells, the animal was male and fertile. In no case was evidence for hermaphroditism observed in the adult animals.

Chimaeras 1210 and 1294 were both $X Y(B 6)<->X Y(F V B / N$ sys/sys). Such animals were of particular interest, as their 


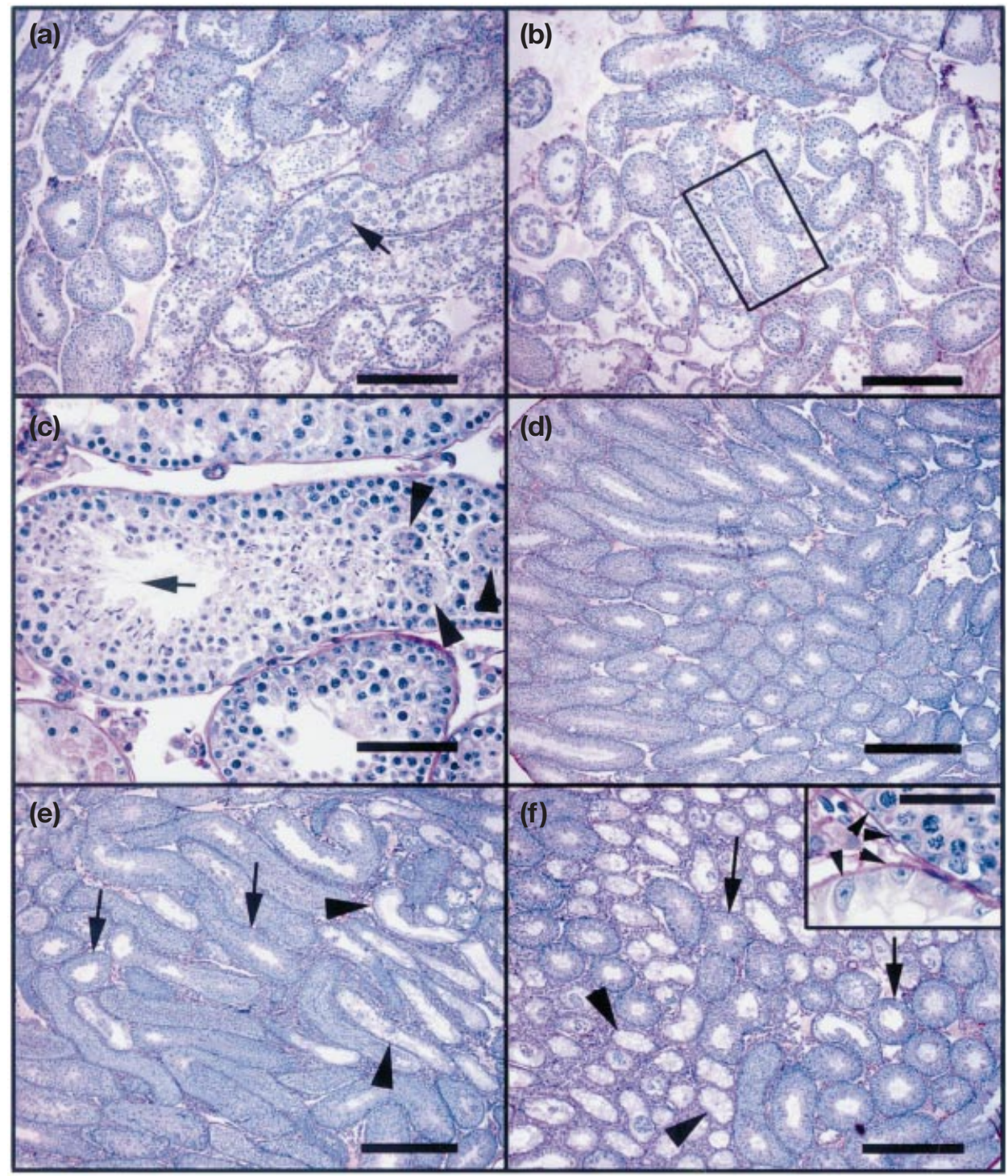

Fig. 3. Testis histology from $\mathrm{FVB} / \mathrm{N}(\mathrm{sys})<->\mathrm{B} 6$ chimaeric mice. (a) Testis histology of chimaera 1294 (B6 XY<->XY FVB/N (sys/sys). Note the presence of symplasts of spermatids (arrow indicates an example), indicative of a sys mutant phenotype, in most tubules. Staining was with periodic acid-Schiff-haematoxylin. (b) Testis histology of chimaera 1210 (B6 XY<->XY FVB/N (sys/sys)). As in panel (a), symplasts of spermatids are observed in most tubules. A tubule containing a mixture of symplasts and normally developing elongate germ cells is boxed. (c) Higher magnification of box in (b). Symplasts of spermatids (arrowheads) and normally developing elongate spermatids (arrow) are present in the same tubule. (d) Testis histology of chimaera 1208 (B6 XX<->XY FVB/N (sys/+)), showing normal spermatogenesis in all tubules. (e) Testis histology of chimaera 1145 (B6 XY<->XX $\mathrm{FVB} / \mathrm{N}($ sys/+)), showing both aspermatogenic (arrowheads) and tubules with normal seminiferous epithelium (arrows). (f) Testis histology of chimaera 1204 (B6 XY<->XX FVB/N (sys/+)). The majority of tubules are aspermatogenic (arrowheads) with relatively few tubules with normal seminiferous epithelium (arrows). Inset: Similar morphology of Sertoli cells (arrowheads) in tubules containing (upper), or lacking (lower) germ cells. Scale bars represent (a,b) $200 \mu \mathrm{m}$, (c) $50 \mu \mathrm{m}$, (d-f) $400 \mu \mathrm{m}$ and (inset in (f) $30 \mu \mathrm{m}$.

testes were predicted to contain a mixture of $\mathrm{B} 6$ and $\mathrm{FVB} / \mathrm{N}$ (sys/sys) germ cells. Despite this prediction, examination of testis histology from these animals revealed an almost exclusive presence of developing germ cells with a sys mutant phenotype (Fig. 3a,b). However, analysis of multiple sections of testis from chimaera 1210 revealed a small number of tubules that contained a mixture of germ cells with either a normal or sys mutant phenotype (Fig. 3c). All of the remaining $X Y<->X Y$ animals exhibited normal gross testis histology. Examination of testis histology from 

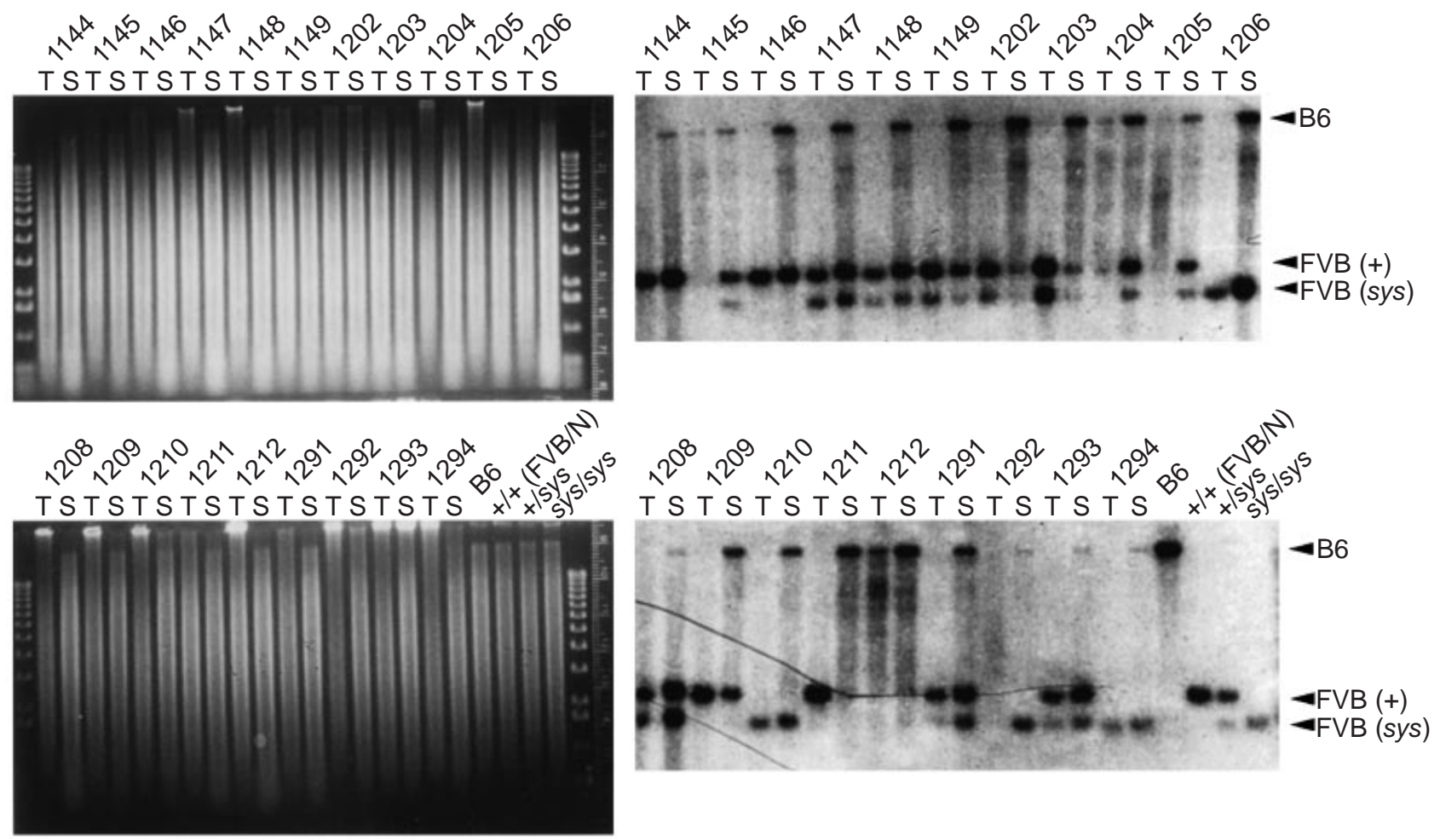

Fig. 4. Molecular genetic analysis of cellular composition of testis and spleen in male $\mathrm{FVB} / \mathrm{N}<->\mathrm{B} 6$ chimaeric mice. Southern blot analysis of genomic DNA extracted from testis (T) or spleen (S) from the individual (numbered) chimaeras, digested with Taq I and hybridized with the insert from pZap1, which identifies RFLPs that discriminate between B6 (14 kb; B6), the wild-type allele of FVB/N $(2.3 \mathrm{~kb} ; \mathrm{FVB}(+))$, and the sys allele of FVB/N $(0.9 \mathrm{~kb} ; \mathrm{FVB}(s y s))$. Control DNA samples are shown on the right side of the lower blot. Photographs of the ethidium bromide-stained gels are shown on the left of each blot to indicate loading of DNA. In some lanes, an expected signal for the B6 allele (14 kb) cannot be clearly observed (for example, testis lane for chimaeras 1205 and 1292 ).

chimaera 1206 (B6 XX<->XY FVB sys/sys) revealed that the seminiferous epithelium was exclusively sys mutant in nature (data not shown).

The number of aspermatogenic seminiferous tubules (that is, lacking germ cells) was quantified for each chimaera, excluding those with a sys/sys component (Table 1). Aspermatogenic tubules were found in one of nine $X Y<->X Y$ chimaeras $(2.6 \%$, in chimaera 1291$)$ but in none of three

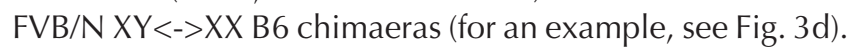
In contrast, analysis of histology from all four B6 $X Y<->X X$ FVB chimaeras revealed aspermatogenic tubules in each animal, with two chimaeras having a large proportion of these $(34.4 \%$, chimaera 1145 , Fig. 3e; $73.4 \%$, chimaera 1204 , Fig. 3f). Sertoli cells with a normal appearance were present within the aspermatogenic seminiferous tubules (Fig. 3f, inset). Aspermatogenic tubules occasionally contained elongate spermatozoa, which had probably originated within adjacent regions of the tubule that had normal spermatogenesis.

\section{Molecular analysis of somatic and germline tissues from chimaeras}

The relative contribution of each donor embryo to cells within the testis of chimaeras was determined by Southern blot analysis with the same DNA probe used to genotype at the sys locus (Fig. 4). As a control for a somatic tissue, DNA was also extracted from the spleen and the results were compared with those obtained from analysis of the tail DNA. Good direct correlation was found between the relative proportion of $\mathrm{B} 6$ and $\mathrm{FVB} / \mathrm{N}$ DNA in tail and spleen. These results also correlated with the estimate for somatic chimaerism deduced from coat colour. For example, on the basis of coat colour, chimaera $1202(\mathrm{XY}<->\mathrm{XY})$ had predominantly B6 contribution to somatic tissues (Fig. 1), which was reflected by the hybridization signals for the B6derived alleles at the sys and Zfy 1 loci in the Southern blot analyses (Fig. 2a,b).

Conversely, on the basis of its mainly albino coat (Fig. 1), chimaera $1210(\mathrm{XY}<->\mathrm{XY})$ had a large proportion of the $\mathrm{FVB} / \mathrm{N}$ component, and this conclusion was supported by the Southern blot analysis of DNA from somatic tissues (Fig. $2 a, b)$.

In contrast, Southern blot analysis of testicular DNA from the $X Y<->X Y$ chimaeras revealed a marked bias towards the FVB/N component (Fig. 4). In each case, a hybridization signal for the B6 allele was observed in the spleen DNA. However, only the $\mathrm{FVB} / \mathrm{N}$-derived hybridization signal was observed in the testis sample from the same chimaera. Thus, 
irrespective of whether balanced somatic chimaerism was evident, there appeared to be an extreme bias in favour of $\mathrm{FVB} / \mathrm{N}$ cells in the testes of these animals.

As expected, in $X X<->X Y$ male chimaeras, the Southern blot hybridization pattern was biased towards the $X Y$ component. For example, chimaeras 1144, 1206, 1208 and 1293, in which the XY embryo was $F V B / N$, displayed a FVB/N pattern only within the testis sample. Similarly, in chimaeras 1145, 1204 and 1212, which each had an XY B6 component, there was a B6 hybridization pattern only in the testis DNA sample.

\section{Discussion}

These results reveal a striking bias in the germ line of B6 $X Y<->X Y$ FVB-aggregation chimaeric mice. Bias in gametic contribution from $X Y<->X Y$ mice of other strain combinations has been reported by Mintz (1968) and Krzanowska et al. (1991). However, the bias observed in the present study is the most extreme example reported to date. Competition among mature spermatozoa can influence gametic contribution in mice (Krzanowska et al., 1991). This mechanism is unlikely to be involved in the present study, as Southern blot analyses indicated that the genetic composition of the testis in B6 XY $<->X Y$ FVB mice is predominantly $F V B$ in origin. A more plausible mechanism would involve a proliferative advantage of FVB germ cells at some stage during germ-line development.

Germ-line development in mice commences with specification of primordial germ cells (PGCs) from the proximal epiblast just before the onset of gastrulation (Lawson and Hage, 1994; Tam and Zhou, 1996). Subsequently the PGCs translocate to the developing hindgut endoderm. Between day 9.0 and day 9.5 of embryo development they leave the gut and migrate into the developing genital ridge between day 10.0 and day 10.5 of embryo development (Molyneaux et al., 2001). In males, gonadal differentiation commences after expression of Sry in the pre-Sertoli cell lineage beginning at day 10.5, and the earliest morphological signs of testicular development are observed by day 12.5 (Capel, 2000). The PGCs differentiate into prospermatogonia, which briefly undergo mitosis before becoming arrested by day 13.5. Prospermatogonial mitosis resumes by day 3 after birth, with formation of spermatogonia by day 5 and commencement of meiosis between day 8 and day 10 after birth (Nebel et al., 1961).

Analysis of testis histology in male B6 XY<->XX FVB chimaeras indicates that competition in germ-cell development may occur during embryogenesis. A significant number of aspermatogenic seminiferous tubules was not observed in $\mathrm{B} 6 \mathrm{XX}<->\mathrm{XY} F \mathrm{FB}$ or $\mathrm{XY}<->\mathrm{XY}$ chimaeric mice. In contrast, two independent B6 $X Y<->X X$ FVB males displayed a high incidence of seminiferous tubules that lacked germ cells. Two X chromosomes are incompatible with male germ cell development, and XX prospermatogonia die shortly after birth (for a review, see McLaren, 1983). Thus, the large number of aspermatogenic tubules in
B6 $X Y<->X X$ FVB males may have arisen during embryogenesis, because of a proliferative advantage of FVB $X X$ PGCs or prospermatogonia that subsequently died after birth. Differences in the rate of proliferation of PGCs during embryogenesis have been observed in $\mathrm{KE}$ and $\mathrm{CBA} / \mathrm{Kw}$ mice, which also displayed bias of the germ line as $X Y<->X Y$ chimaeras (Krzanowska et al., 1991; Wabik-Sliz et al., 1998). Aspermatogenic tubules might also have arisen as a consequence of excessive numbers of $X X$ Leydig cells or Sertoli cells. However, $X X$ cells in $X X<->X Y$-aggregation chimaeras can form Leydig cells with a normal appearance that are capable of supporting spermatogenesis in adjacent tubules (Burgoyne et al., 1988). In addition, genetic analysis (Mazeyrat et al., 2001) has indicated that adult mouse spermatogenesis can be supported (at least up to the first meiotic metaphase) by $X X$ Leydig cells that express the Y-linked gene Eif2s3y. Thus, the presence of two $X$ chromosomes is not incompatible with Leydig-cell function and Eif2s3y is formally the only Y-linked gene the expression of which in Leydig cells might be required for adult spermatogenesis (at least until the first meiotic metaphase). Sertoli cells in adult $X Y<->X X$ chimaeric mice of different strain combinations are almost exclusively (>90\%) XY in origin (Palmer and Burgoyne, 1991; Patek et al., 1991). On the basis of these findings, the aspermatogenic tubules are unlikely to arise as a consequence of a large number of XX Sertoli cells.

Ultimately, further studies are required to identify the precise stage(s) in development at which the bias in germline development occurs in B6<->FVB chimaeras and the basis for the advantage of FVB male germ cells compared with B6 male germ cells. Development of female germ cells in these chimaeras, which although less biased, was still evident, must also be examined. Such studies could be facilitated by use of ROSA26.B6 mice (Friedrich and Soriano, 1991), the cells of which are marked by $\beta$-galactosidase expression, enabling single-cell resolution of the parental origin of both somatic and germ cells in the developing gonad.

Testes from B6 XX or $\mathrm{XY}<->\mathrm{XY}$ FVB sys/sys chimaeras contained essentially only germ cells with a sys-mutant phenotype, indicating that the wild-type B6 component of somatic tissues was unable to rescue the germ-cell defect. This finding indicates that if the defect in sys mice is germcell non-autonomous, it is likely to affect a type of somatic cell within the testis. The fact that the germ line in $X Y<->X Y$ chimaeras was almost completely derived from the FVB component, and that the testis histology of B6 $X Y<->X Y$ FVB (sys/sys) was almost exclusively sys mutant, is consistent with the contention that the sys defect is germ-cell autonomous. However, if the germ-cell defect is an indirect effect of defective Sertoli (or other somatic) cells within the testis, and a similar bias exists in the somatic-cell component of the testis of B6 $X Y<->X Y$ FVB mice, this could produce the same experimental outcome. Whether the haploid germ-cell defect in sys mutant mice is germ-cell autonomous remains unclear. This issue should be resolved by a more direct contemporary experimental method, 
involving use of germ-cell transplantation in conjunction with cell lineage markers afforded by ROSA26 mice (Friedrich and Soriano, 1991; Brinster and Avarbock, 1994; McLean et al., 2001).

The $\mathrm{FVB} / \mathrm{N}$ strain is commonly used for production of random integration transgenic mice (Taketo et al., 1991). A strategy of using $\mathrm{C} 57 \mathrm{BL} / 6$ mice as a partner for $\mathrm{FVB} / \mathrm{N}$ in a chimaeric analysis is attractive due to its common use, and the differences in pigmentation and genetic polymorphism associated with the different origins of the $Y$ chromosome in these strains. The results of this study indicate that this choice of strain combination is unsuitable when the experimental design requires generation of a balanced male, or female, germ line.

This work was conducted while the author was a research associate in the laboratory of Paul Overbeek at Baylor College of Medicine, Houston, TX. The author thanks Paul Overbeek for support, Gerri Hanten and Paul Overbeek for assistance with production of aggregation chimaeric mice, David Page for DNA probes and Steven L'Hernault for constructive criticism of the manuscript.

\section{References}

Ausubel FM, Brent R, Kingston RE, Moore DD, Seidman JG, Smith JA and Struhl K (1994) Current Protocols in Molecular Biology John Wiley \& Sons, Inc., New York

Brinster RL and Avarbock MR (1994) Germline transmission of donor haplotype following spermatogonial transplantation Proceedings National Academy of Sciences USA 9111 303-11 307

Burgoyne PS (1975) Sperm phenotype and its relationship to somatic and germ line genotype: a study using mouse aggregation chimaeras Developmental Biology 44 63-76

Burgoyne PS, Buehr M, Koopman P, Rossant J and McLaren A (1988) Cellautonomous action of the testis-determining gene: Sertoli cells are exclusively $X Y$ in $X X-X Y$ chimaeric mouse testes Development 102 $443-450$

Capel B (2000) The battle of the sexes Mechanisms of Development 92 89-103

Friedrich G and Soriano P (1991) Promoter traps in embryonic stem cells: a genetic screen to identify and mutate developmental genes in mice Genes and Development 5 1513-1523

Gearhart J and Oster-Granite ML (1981) Reproduction in a population of chimaeric mice: relationship of chromosomal sex to functional germ cells and proportions of chimaeric components in several tissues Biology of Reproduction 24 713-722

Handel MA, Washburn LL, Rosenberg MP and Eicher EM (1987) Male sterility caused by $p^{6 H}$ and $q k$ mutations is not corrected in chimaeric mice Journal of Experimental Zoology 243 81-92

Hogan BLM, Beddington RSP, Costantini F and Lacy E (1994) Manipulating the Mouse Embryo: a Laboratory Manual Cold Spring Harbor Laboratory Press, Cold Spring Harbor, NY

Krzanowska H, Wabik-Sliz B and Rafinski J (1991) Phenotype and fertilizing capacity of spermatozoa of chimaeric mice produced from two strains that differ in sperm quality Journal of Reproduction and Fertility 91 667-676

Lawson K and Hage W (1994) Clonal analysis of the origin of primordial germ cells in the mouse Ciba Foundation Symposium 182 68-91

MacGregor GR, Russell LD, VanBeek MEA, Hanten GR, Kovac MJ, Kozak CA, Meistrich ML and Overbeek PA (1990) Symplastic spermatids (sys): a recessive insertional mutation in mice causing a defect in spermatogenesis Proceedings National Academy of Sciences USA 87 5016-5020

McLaren A (1975) Sex chimaerism and germ cell distribution in a series of chimaeric mice Journal of Embryology and Experimental Morphology 33 205-216

McLaren A (1983) Does the chromosomal sex of a mouse germ cell affect its development? In Current Problems in Germ Cell Differentiation pp 225-240 Eds A McLaren and CC Wylie. Cambridge University Press, Cambridge

McLean DJ, Johnston DS, Russell LD and Griswold MD (2001) Germ cell transplantation and the study of testicular function Trends in Endocrinology and Metabolism 12 16-21

Mardon G, Mosher R, Disteche CM, Nishioka Y, McLaren A and Page DC (1989) Duplication, deletion, and polymorphism in the sex-determining region of the mouse $Y$ chromosome Science 243 78-80

Mazeyrat S, Saut N, Grigoriev V, Mahadevaiah SK, Ojarikre OA, Rattigan A, Bishop C, Eicher EM, Mitchell MJ and Burgoyne PS (2001) A Yencoded subunit of the translation initiation factor Eif2 is essential for mouse spermatogenesis Nature Genetics 29 49-53

Mintz B (1964) Formation of genotypically mosaic mouse embryos and

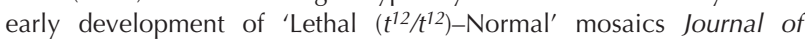
Experimental Zoology 157 273-292

Mintz B (1968) Hermaphroditism, sex chromosomal mosaicism and germ cell selection in allophenic mice Journal of Animal Science 27 51-60

Mintz B and Palm J (1969) Gene control of hematopoiesis. I. Erythrocyte mosaicism and permanent immunological tolerance in allophenic mice Journal of Experimental Medicine 129 1013-1027

Molyneaux KA, Stallock J, Schaible K and Wylie CC (2001) Time-lapse analysis of living mouse germ cell migration Developmental Biology 240 488-498

Mystkowska ET and Tarkowski AK (1968) Observations on CBA-p-CBAT6T6 mouse chimaeras Journal of Embryology and Experimental Morphology 20 33-52

Nebel BR, Mamarose AP and Hackett EM (1961) Calendar of gametic development in the prepuberal male mouse Science 134 832-833

Palmer SJ and Burgoyne PS (1991) In situ analysis of fetal, prepuberal and adult $\mathrm{XX}-\mathrm{XY}$ chimaeric mouse testes: Sertoli cells are predominantly, but not exclusively, XY Development 112 265-268

Patek CE, Kerr JB, Gosden RG, Jones KW, Hardy K, Muggleton-Harris AL, Handyside AH, Whittingham DG and Hooper ML (1991) Sex chimaerism, fertility and sex determination in the mouse Development $113311-325$

Rossant J and Spence A (1998) Chimaeras and mosaics in mouse mutant analysis Trends in Genetics 14 358-363

Russell LD, Sinha Hikim AP, Overbeek PA and MacGregor GR (1991) Testis structure in the sys (symplastic spermatids) mouse American Journal of Anatomy 192 169-182

Taketo M, Schroeder AC, Mobraaten LE et al. (1991) FVB/N: an inbred mouse strain preferable for transgenic analyses Proceedings National Academy of Sciences USA 88 2065-2069

Tam PP and Zhou SX (1996) The allocation of epiblast cells to ectodermal and germ-line lineages is influenced by the position of the cells in the gastrulating mouse embryo Developmental Biology 178 124-132

Tarkowski AK (1961) Mouse chimaeras developed from fused eggs Nature $190857-860$

Wabik-Sliz B, Paczuska I, Wisniewska A and Lenartowicz M (1998) The comparison of primordial germ cell populations in embryos of KE and CBA inbred strains of mice Folia Histochemica et Cytobiologica 36 $81-85$

Received 11 February 2002.

First decision 9 April 2002.

Revised manuscript received 23 May 2002.

Accepted 10 June 2002. 